Int. J. Dev. Biol. 60: 245-254 (2016)

doi: $10.1387 / \mathrm{ijdb} .160155 \mathrm{tl}$

\title{
The master Greatwall kinase, a critical regulator of mitosis and meiosis
}

\author{
SUZANNE VIGNERON, PERLE ROBERT, KHALED HACHED, LENA SUNDERMANN, SOPHIE CHARRASSE, \\ JEAN-CLAUDE LABBÉ, ANNA CASTRO* and THIERRY LORCA* \\ Equipe Labellisée Ligue Contre le Cancer. Université de Montpellier, \\ Centre de Recherche de Biologie Cellulaire de Montpellier, CNRS UMR 5237, France
}

\begin{abstract}
Entry into mitosis requires the coordinated activation of various protein kinases and phosphatases that together activate sequential signaling pathways allowing entry, progression and exit of mitosis. The limiting step is thought to be the activation of the mitotic Cdk1-cyclin B kinase. However, this model has recently evolved with new data showing that in addition to the Cdk1-cyclin B complex, Greatwall (Gwl) kinase is also required to enter into and maintain mitosis. This new concept proposes that entry into mitosis is now based on the combined activation of both kinases Cdk1-cyclin B and Gwl, the former promoting massive phosphorylation of mitotic substrates and the latter inhibiting PP2A-B55 phosphatase responsible for dephosphorylation of these substrates. Activated Gwl phosphorylates both Arpp19 and ENSA, which associate and inhibit PP2A-B55. This pathway seems relatively well conserved from yeast to humans, although some differences appear based on models or techniques used. While Gwl is activated by phosphorylation, its inactivation requires dephosphorylation of critical residues. Several phosphatases such as PP1, PP2A-B55 and FCP1 are required to control the dephosphorylation and inactivation of Gwl and a properly regulated mitotic exit. Gwl has also been reported to be involved in cancer processes and DNA damage recovery. These new findings support the idea that the Gwl-Arpp19/ENSA-PP2A-B55 pathway is essential to achieve an efficient division of cells and to maintain genomic stability.
\end{abstract}

KEY WORDS: Greatwall, Arpp19, ENSA, mitosis, meiosis

\section{Auto amplification loop of $M$ phase promoting factor (MPF)}

Progression from the G2 phase to the end of mitosis is governed by the sequential phosphorylation and dephosphorylation of different mitotic regulators.

Entry into mitosis is controlled by a tight network of proteins that regulate activation of the crucial regulator of mitosis: Cdk1-cyclin B. Once activated, Cdk1-cyclin B triggers mitotic entry by phosphorylating target proteins promoting nuclear envelope breakdown (NEB), chromosome condensation, microtubules dynamics, spindle assembly and the separation of sister chromatids. On the other hand, exit from mitosis is triggered by the APC/C (Anaphase Promoting Complex/Cyclosome)-dependent ubiquitination and degradation of cyclin B resulting in the inactivation of Cdk1-cyclin B kinase (Fig. 1). Like many protein kinases, a balance of phosphorylation and dephosphorylation regulates Cdk1-Cyclin B activation. First, once bound to cyclin B, CAK (Cdk-Activating-Kinase) phosphorylates
Cdk1 on the key residue Thr 161 (Activation loop) and stabilizes the Cdk1-cyclin B association allowing the functional conformation required for kinase activity. Second, the balance between Wee1/ Myt1 kinases and Cdc25 phosphatase regulates Cdk1-cyclinB activity. Wee1 and Myt1 kinases phosphorylate Thr14 and Tyr15 (ATP-bindind pocket) and thereby inactivate Cdk1 bound to cyclin B. At the G2-Mitosis transition, Cdc25 phosphatase is responsible for the dephosphorylation of Cdk1 on its two inhibitory sites Thr14 and Tyr15, thus allowing activation of the Cdk1-cyclin B kinase and initiation of mitosis. The unanimously accepted model proposes that at the end of the G2 phase, dephosphorylation of Thr14 and Tyr15 of Cdk1 by Cdc25 induces initial activation of Cdk1-cyclin B complex, which in turn phosphorylates Cdc25, Wee1 and Myt1, activating the Cdc25 phosphatase and inactivating Myt1 and Wee1 protein kinases. These rapid events promote full activation

Abbreviations used in this paper: Gwl, greatwall; MPF, M phase promoting factor; RNAi, RNA interference.

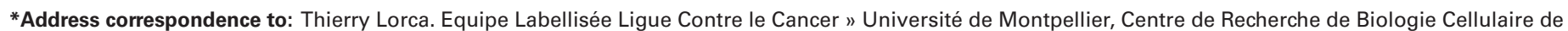
Montpellier, CNRS UMR 5237, France. e-mail: thierry.lorca@crbm.cnrs.fr
}

Accepted: 19 May 2016.

ISSN: Online 1696-3547, Print 0214-6282 
Fig. 1. Kinases and phosphatases control entry into mitosis. Mitotic entry requires the coordinated activation of kinases and phosphatases that together activate sequential pathways allowing mitotic entry and exit. Exit of mitosis is triggered by degradation of cyclin $B$ that promotes Cdk1-cyclin B inactivation.

of Cdk1-cyclin B kinase and drives irreversible mitotic entry. This mechanism is commonly called auto amplification loop of MPF (M Phase Promoting Factor) (Kishimoto, 2015; Lindqvist et al., 2009; Castro et al., 2005).

\section{Transcriptional control of Cyclin B}

In addition to the regulation of the amplification loop of MPF, the transcription of cyclin B is another key factor that controls the timing of mitotic entry. Transcription of cyclin B starts during Sphase, peaking in G2 phase and persists until the end of mitosis. Cyclin B is thought to be a main target of the transcription factor FoxM1 at the G2-M transition. Consistent with the critical threshold of cyclin B concentration required for entry into mitosis, FoxM1deficient cells delay in G2. These defects are partially rescued upon overexpression of cyclin B1 (Laoukili et al., 2005). Transcription factors involved in cyclin B transcription can themselves be positively controlled by the Cdk-cyclin activity. However, during the G2 phase, the activity of Cdk1-cyclin B kinase is low and it is hardly conceivable that it may regulate the activity of FoxM1. Cdk2-cyclin $\mathrm{E}$ and Cdk1/2-cyclin A kinases are probably more likely to promote this transcriptional activation (Laoukili et al., 2008).

\section{Polo-like kinase family and Cdk-Cyclin A}

While the opposing activities of Wee1/Myt1 and Cdc25 directly regulate the activation of $\mathrm{Cdk1-cyclin} B$ kinase, the polo-like kinase (PIk1 and Plx1 in human cells and in Xenopus respectively) is part of the positive-feedback loop. Both PIk1 and PIx1 phosphorylate and activate Cdc25 at the G2/M-phase transition (Kumagai and Dunphy, 1996; Roshak et al., 2000). In turn, Cdk1-cyclin B promotes a positive feedback allowing activation of Plx1 (Abrieu et al., 1998). Besides the role of Cdk1-cyclin B kinase, other critical activation mechanism of Plk1 has been recently described in mammalian cells by two independent laboratories. At mitotic entry, the Aurora-A kinase phosphorylates Plk-1 on the activating residue, T210. This activation is enhanced by the protein Bora through its binding to Aurora A and/or Plk1 kinases (Macurek et al., 2008; Seki et al., 2008). In turn, Plk1 promotes the proteolysis of Bora. Although most Bora protein is degraded when cells enter into mitosis, this pathway is partially maintained active when cells progress through mitosis. This suggests that only a small stabilized fraction of Bora is required to maintain Plk1 activity throughout mitosis (Bruinsma et al., 2014; Chan et al., 2008; Seki et al., 2008).

Besides Plk1/Aurora-Apathway, other lines of evidence support a role of Cdk-cyclin A at the first step of mitotic entry. First, the kinase activity associated with cyclin $A$ increases before the one associated with cyclin B (Goldstone et al., 2001). Next, experiments on Drosophila cyclin A mutants, show that in the absence of cyclin A,

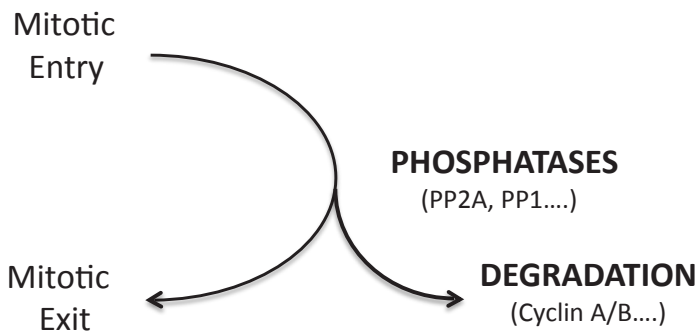

cells arrest in the G2 phase (Knoblich and Lehner, 1993). On the other hand, embryonic cells from mice lacking cyclin A enter into mitosis with a small delay in G2. In cells devoid of cyclin A, cyclin $E$ expression is prolonged and may compensate the loss of cyclin A (Kalaszczynska et al., 2009). However, inhibition of Cdk-cyclin A with a N-terminal portion of the p21 Cip1 inhibitor prevents HeLa cells from entering mitosis. Moreover, if the inhibition of Cdk2-cyclin A occurs in early prophase it induces a return of the treated cells to G2 phase (Furuno et al., 1999).

Further evidence supporting a role of cyclin A in promoting mitotic entry comes from experiments performed in human cells. Cdk2-cyclin A kinase activity is required to activate Cdc25 phosphatase, leading to the dephosphorylation of Cdk1 on Tyr15 and initiation of mitotic entry. Down-regulation of cyclin A by RNA interference (RNAi) or inhibition of Cdk2-cyclin A kinase using a dominant-negative mutant of Cdk2, prevents activation of Cdc25 B/C phosphatases and dephosphorylation of Tyr 15 on Cdk1, and the subsequent Cdk1-cyclin B activation (Mitra and Enders, 2004). Besides Cdc25 activation, it has been proposed that Cdk2-cyclin A may also initiate the inhibition of Wee1 directly by a negative phosphorylation, thereby allowing activation of a fraction of Cdk1 cyclin B complex (Fung et al., 2007; Li et al., 2010; Deibler and Kirschner, 2010). Collectively, these data indicate that the levels of cyclin B expressed in cells are insufficient for initiation of mitotic entry but requires a combination of regulatory components (Fig. 2).

\section{Greatwall (Gwl) kinase: the critical regulator of PP2A-B55}

Although, for a long time, scientists have devoted their time to understand how protein kinases were involved in the mechanisms controlling mitotic entry, the identity of the phosphatases that abolish these kinase-dependent phosphorylations remained elusive. Recently, two independent studies carried out in Xenopus egg extracts, highlighted the critical role of protein phosphatase 2A in mitotic entry (Mochida et al., 2009; Vigneron et al., 2009). PP2A phosphatase is a trimeric complex consisting of a catalytic subunit, a scaffold subunit $A$ and a regulatory subunit $B$ that belong to the gene family B: B (B55), B'(B56), B"(PR72) and B '"(PR93) and that is thought to be responsible for the substrate specificity of PP2A (Shi, 2009).

One of these two works showed that the activity of PP2A was high in interphase and low during mitosis suggesting a tight regulation of the activity of this phosphatase at the G2-M transition (Mochida et al., 2009). In this study, depletion of PP2A-B55 delta isoform from interphase egg extracts promoted activation of Cdk1-cyclin $B$ kinase, phosphorylation of mitotic substrates and entry into mitosis (Mochida et al., 2009). Meanwhile, the other study demonstrated the involvement of Greatwall (Gwl) in controlling PP2A activity (Vigneron et al., 2009). This story relied on experiments in which 
Gwl was specifically depleted from metaphase II arrested egg extracts (meiotic M-phase or CSF), extracts where Cdk1-cyclin $B$ activity is naturally high and constant. This depletion promoted mitotic substrate dephosphorylation and "mitotic exit" even in the presence of a high Cdk1 activity. Strikingly, inhibition or depletion of PP2A-B55 in Gwl-devoid CSF extracts rescued Cdk1-cyclinB substrate dephosphorylation and restored mitosis (Vigneron et al., 2009; Castilho et al., 2009).

How has the link between Gwl and PP2A-B55 been uncovered. The first insights on the role of Gwl kinase were found in Drosophila in which Gwl mutant cells displayed a delay in mitotic entry as well as in metaphase, with spindle aberrations and chromosome segregation defects (Bettencourt-Dias et al., 2004; Yu et al., 2004). These finding indicated that Gwl kinase might be crucial for getting into and progressing through mitosis. By using Xenopus egg extracts, Goldberg's group showed that Gwl was activated in metaphase II, and its depletion from CSF extracts promoted metaphase II exit. Interestingly, exit was not accompanied with degradation of cyclin B excluding the role of APC/C in CSF exit. In the same line, cycling egg extracts devoid of Gwl were prevented to enter into mitosis and accumulated with inhibitory phosphorylated Cdk1 on Tyr15 (Yu et al., 2006). These effects are rescued by overexpression of constitutively active Cdk1AF (Thr14A and Tyr15F Cdk1 mutant).

So, how Gwl maintains metaphase II arrest and allows entry into mitosis? The first hypothesis was that Gwl could promote Cdc25 activation and thus promote tyrosine dephosphorylation of Cdk1. In this line, the addition of an amount of active Gwl able to promote mitotic entry in untreated egg extracts induced partial phosphorylation of Cdc25 in extracts in which roscovitine was added to inhibit Cdk1-Cyclin B and cycloheximide was supplemented to prevent protein synthesis. These data suggest that $\mathrm{Gwl}$ is involved in the activation of Cdc25. Although Cdc25 could be a very promising

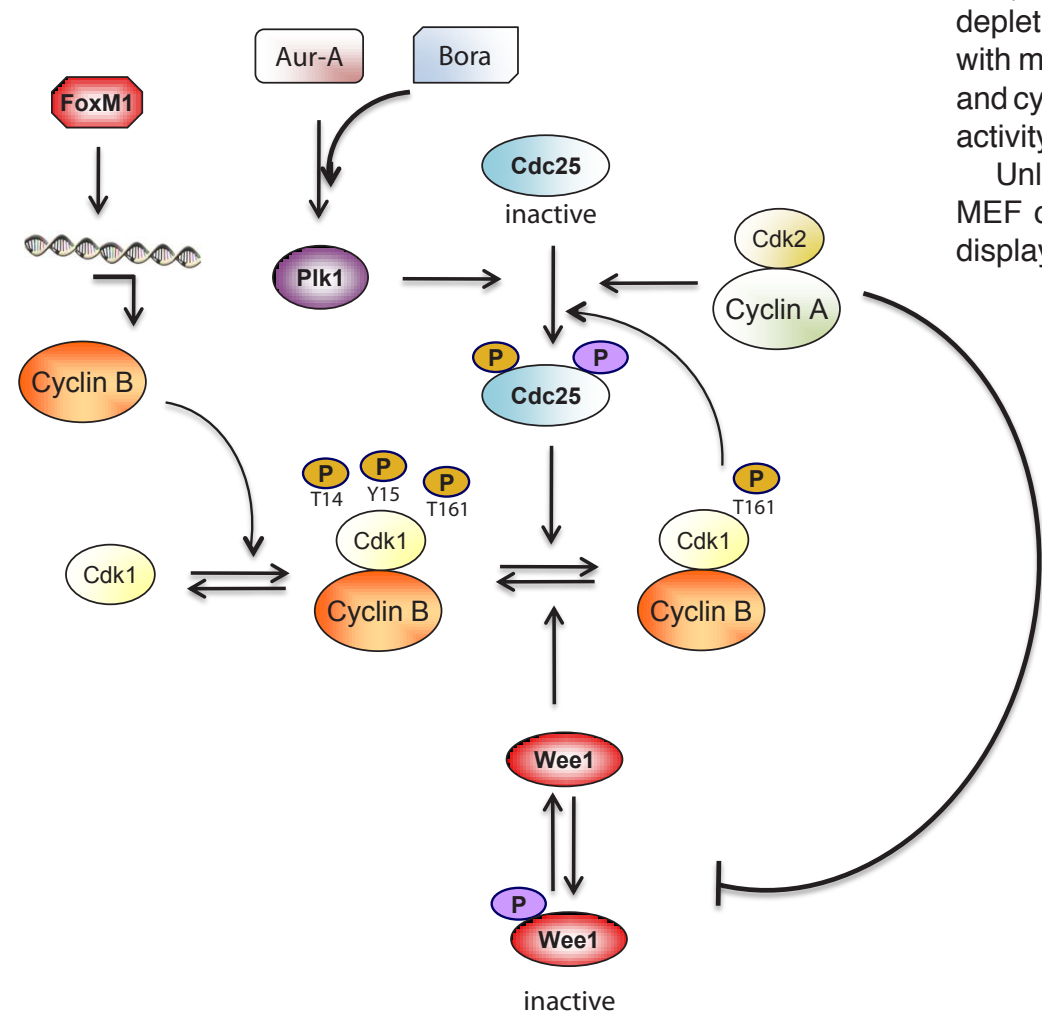

target due to its involvement in promoting activation of Cdk1-cyclin $\mathrm{B}$, further studies demonstrated that this phosphorylation is not directly due to Gwl (Zhao et al., 2008). However, taking advantage of the fact that Xenopus eggs only contain the Cdc25C isoform, a study from our lab showed that depletion of Cdc25C from CSF extracts promotes metaphase II exit with a decrease of Cdk1-cyclin $B$ activity but without degradation of cyclin B. According to what had previously been described for Gwl depletion, metaphase Il arrested Xenopus oocytes microinjected with anti-Cdc25C antibodies or downregulation of Cdc25A using double-stranded RNA in mouse oocytes displayed dephosphorylation of mitotic substrates and Cdk1-cyclin B inactivation, but did not affect the levels of cyclin B (Lorca et al., 2010, Oh et al., 2013). To clarify this molecular mechanism, co-depletion of Cdc25C and Wee1 was performed in CSF extracts. Results from this experiment showed that these depletions rescued the phenotype induced by Cdc25C removal, suggesting that the regulatory balance of the Cdc25 phosphatase and the Wee 1 kinase is maintained active throughout the cell cycle (Lorca et al., 2010). In this line, we prevented inactivation of Cdk1cyclin B by Wee1 depletion in CSF extracts, removal of Gwl still induced M-phase exitand mitotic substrate dephosphorylation even in the presence of high Cdk1-cyclin B activity (Vigneron etal., 2009). Interestingly, under these conditions the removal of PP2Acorrected the Gwl-dependent defects thereby maintaining metaphase II arrest (Vigneron et al., 2009). Results obtained by two independent groups further identified PP2A-B55/Twins (Xenopusand Drosophila respectively) as the phosphatase targeted by Gwl (Castillo et al., 2009; Wang et al., 2011). These results led to propose that mitotic entry and progression were mediated by both activation of Cdk1cyclin B and inhibition of PP2A by Gwl (Vigneron et al., 2009). This model is supported by recent studies in mammalian cells in which Gwl knockdown by RNAi strongly delays or arrests cells in G2 (Burgess et al., 2010; Voets et al., 2010). However, when Gwl depletion is not complete, cells enter and progress through mitosis with multiple defects such as binucleation, DNA bridges formation and cytokinesis failure. These defects were likely due to high PP2A activity, as inhibition of PP2A rescued these mitotic entry defects.

Unlike results in Gwl knockdown mammalian cells, Gwl knockout MEF cells enter into mitosis with normal kinetics although they display similar mitotic defects to cells partially depleted of Gwl by
Fig. 2. Regulation of mitotic entry: transcription and degradation ensure that cyclin B levels peak at the end of the G2-phase and decrease at metaphase/anaphase transition. Translated cyclin $B$ binds to Cdk 1 and this complex is maintained inactive by Wee 1/Myt1 kinases. The Aurora-A kinase phosphorylates and activates Plk-1. This phosphorylation is enhanced by the Bora protein, which binds Aurora-A kinase. Activated PIk-1 and Cdk2-cyclin A phosphorylate and activate some molecules of Cdc25 thereby promoting initial activation and autoregulatory activation of Cdk1-cyclin B kinase. 
siRNA. On the other hand, RNAi-induced knockdown of Gwl in porcine oocytes blocks or delays meiotic maturation. Gwl-deficient oocytes that enter and progress through meiosis displayed defects in chromosome condensation and decreased MPF activity (Li et al., 2013). Interestingly, while the Gwl/PP2A pathway is required for meiosis I entry and/or progression in Xenopus, Drosophila, Porcine and Starfish oocytes (Dupré et al., 2013; Dupré et al., 2014; Hara et al., 2012; Li et al., 2013; Von stetina et al., 2008), Gwl-deficient mouse oocytes can initiate meiosis and progress to metaphase I with normal timing. These oocytes completed meiosis I but with a delay in polar body extrusion and with a perturbed degradation of the APC/C substrates securin and cyclin B1 (Adhikari et al., 2014). According to the role of $\mathrm{Gwl}$ in the control of APC activity, mammalian cells knocked down of Gwl by RNAi, display a delay in cyclin B degradation when cells progress through mitosis, suggesting that $\mathrm{Gwl}$ is required to trigger a full and timely activation of APC/C (Voets and Wolthius, 2015). Although Gwl-null oocytes normally progressed through meiosis I, they failed to reactivate Cdk1-Cyclin B kinase after the polar body extrusion and never entered into metaphase II. This is in agreement with a requirement of $\mathrm{Gwl}$ to maintain metaphase II state in Xenopus oocytes (Yu et al., 2006; Vigneron et al., 2009). Altogether, these data are still confusing because Gwl-PP2A-pathway seems to be necessary at different mitotic stages depending on the method of depletion (knockout or knockdown) that have been used or the species used (mouse or porcine oocytes). Further detailed explorations are required to understand how Gwl controls the meiotic progression and metaphase II arrest.

\section{Gwl controls PP2A-B55 activity through the phosphorylation of Arpp19/ENSA}

While it was clear that Gwl regulates PP2A-B55 activity, the direct phosphorylation of this phosphatase by Gwl had never been observed indicating that Gwl might indirectly inhibit PP2A. Therefore, the molecular mechanism by which Gwl promoted PP2A-B55 inhibition remained unknown.

To explore how Gwl maintains the metaphase II state and controls mitotic entry, two independent groups developed a biochemical strategy using Xenopus interphase egg extracts to characterize the substrates of $\mathrm{Gwl}$ responsible of PP2A-B55 inhibition. Using this approach, they both identified a protein of $20 \mathrm{kD}$ named Arpp19 (cyclic adenosine monophosphate-regulated phosphoprotein 19) sharing homologies with the small protein $\alpha$-Endosulfine (ENSA) (Gharbi-Ayachi et al., 2010; Mochida et al., 2010). Both Arpp19 and ENSA are phosphorylated in vitro by an active form of Gwl at one key residue (Ser62 and Ser67 of human Arpp19 and human ENSA respectively). Once phosphorylated, these two proteins associate with and inhibit the activity of PP2A-B55 phosphatase. In addition, overexpression of these phosphorylated substrates in interphase Xenopus eggs extracts induced inhibition of PP2A-B55, activation of Cdk1-cyclin B, phosphorylation of mitotic substrates and entry into mitosis. However, as we will see later, the exact involvement of each of these two substrates in vivo remains undefined. While one of the two groups showed that depletion of ENSA affects mitotic entry (Mochida et al., 2010), the second study indicated that no effect was observed on the entry and maintenance of mitosis after the depletion of this protein. In addition, they showed that metaphase II maintenance and mitotic entry in the first embryonic cycle is rather under the control of Arpp19 (Gharbi-Ayachi et al., 2010) (Fig. 3).

Studies in oocytes reported the involvement of these two substrates in the meiotic progression. RNAi-mediated knockdown of ENSA from mouse oocytes results in a block of meiotic maturation at prophase I. However, some of the treated oocytes still undergo normal progression in meiosis I and to metaphase II, suggesting that either depletion of ENSA was not efficient enough, or that endogenous Arpp19 was sufficient to activate the Arpp19/ENSA-Gwl/ PP2A-B55 pathway in these oocytes (Matthews and Evans, 2014). Contrary to mouse oocytes in which both Arpp19 and ENSA appear to participate in the Gwl/PP2A-B55 pathway, in pig oocytes Arpp19 is entirely responsible for $\mathrm{PP} 2 \mathrm{~A}-\mathrm{B} 55$ regulation since ENSA protein is absent in these oocytes ( $\mathrm{Li}$ et al., 2013). Finally, an active Gwl/

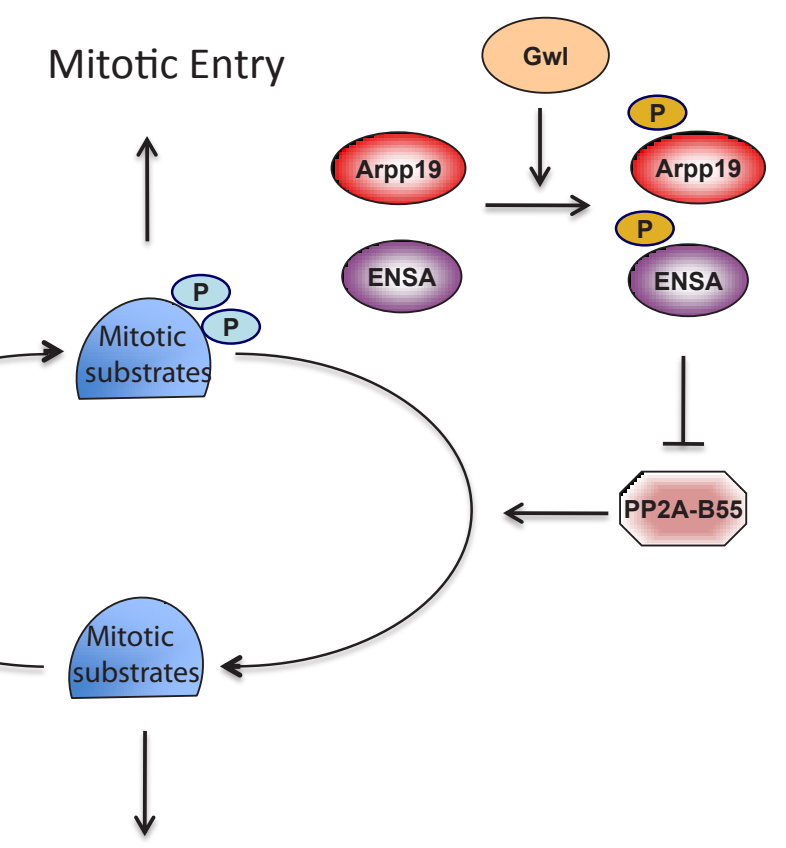

\section{Mitotic Exit}

Fig. 3. Cdk1-cyclin B-Greatwall-PP2AB55 pathway: Cdk1-cyclin B phosphorylates mitotic substrates at mitotic entry counteracted by PP2A-B55 at mitotic exit. Greatwall phosphorylates Arpp19/ENSA inhibitors and thereby blocks PP2A-B55 activity. 
Arpp19/ENSA/PP2AB55 pathway has been studied in mammalian cells. From those studies, the role of Arpp19 could not be determined due to the lack of suitable tools for its detection. However, removal of ENSA in these cells perturbed MPF/PP2A-B55 balance inducing cytokinesis defects, resulting at least in part by abnormal dephosphorylation of PRC1 (Cundell et al., 2013). Further studies will be required to understand the specific roles of each substrate in the Gwl/PP2A-B55 pathway throughout the cell cycle.

The Gwl kinase and its substrates have now emerged in a wide range of organisms. In Drosophila, Endos is the single Arpp19/ ENSA orthologue. Endos is phosphorylated by Gwl on the conserved FDSpGDY motif, a phosphorylation that is essential for its function and that is present in every member of this protein family. Additionally, knockdown of Endos prevents the maintenance of the mitotic state. In starfish oocytes, as well as in Drosophila, only one single homologue of Arpp19/ ENSA is found from database search. The starfish homologue is phosphorylated by both, Gwl and Cdk1-cyclin B kinases at two different sites, Ser106 and Ser69 respectively. Both Ser106 and Ser69 phosphorylations participate to the PP2A-B55-dependent inhibition of Arpp19. On the other hand, S106 phosphorylation of Arpp19/ENSA starfish homologue is required for correct chromosome segregation (Okumura et al., 2014). The dual role of Gwl and Cdk1-cyclin B kinases to directly enable Arpp19/ENSA as mitotic phosphatase inhibitors has not been described in other organisms so far. However, biochemical experiments using thiophosphorylated Xenopus ENSA on both Cdk1-cyclin B and Gwl sites (Thr28 and Ser67 respectively) confirm that Ser67 phosphorylation by $\mathrm{Gwl}$ is the major key residue involved in PP2A-B55 inhibition (Mochida 2014).

Finally, the budding yeast homologues of Arpp19/ENSA called Igo1 and Igo2 are phosphorylated by Rim15 (Gwl) kinase in a cell cycle regulated manner. Phosphorylated Igo1 binds and inhibits PP2A-B55 resulting in the phosphorylation of mitotic substrates when added to Xenopus egg extracts. Moreover, Igo1 can directly inhibit PP2A-Cdc55 (the B55 homologue in budding yeast) activity in vitro, and Igo1/2-deficient cells are defective in mitotic entry into (Juanes et al., 2013). In budding yeast, PP2A-Cdc55 is controlled by its subcellular distribution through the association of the regulatory subunit Cdc55 to both proteins Zds (Zillion different screens) and Igo1/2. Igo1/2 and Zds proteins might share overlapping function. Indeed, Zds are exclusively cytoplasmic contrary to lgo1/2 which are enriched in the nucleus. In this context, overexpression of Zds can sequestrate PP2A-Cdc55 in the cytoplasm and promote precocious mitotic entry. On the other hand, Igo1/2 control nuclear accumulation of PP2A-Cdc55 supporting the idea that Igo1/2 inhibit nuclear PP2A-Cdc55 by direct binding (Bontron et al., 2013; Rossio et al., 2014).

In fission yeast, cell growth is controlled by nutrients, which in turn positively regulate TORC1 pathway to promote growth by enhancing anabolic processes. Yeast cells divide in a size threshold manner that depends on nutriment availability reaching the higher volume before mitosis under rich nitrogen conditions. Conversely, when yeast cells are shifted to a poor nitrogen medium they enter into mitosis with a reduced cell size. A recent study performed in S.pombe showed that the Gwl-Igo-PP2A pathway is controlled by the nutritional environment. In the presence of nutriments, TORC1 inhibits Gwl/PpK18 pathway and maintains high activity of PP2A resulting in large cells in G2 before they undergo mitosis. Conversely, when yeast is grown in nutrient poor medium, TORC1 activity drops thereby activating Gwl/Ppk18, which phosphorylates Igo1 at Ser64, inhibiting PP2A. In such conditions, the inhibition of PP2A promotes a premature activation of Cdk-cyclin complex producing smaller mitotic cells indicating that this pathway can be activated under specific environmental events (Chica et al., 2016).

\section{Activation and inactivation of Gwl}

Gwl activity is tightly controlled during mitotic entry, progression and exit. Gwl is phosphorylated at mitotic entry, thereby leading to its activation. In vitro phosphorylation of Gwl by MPF induces partial activation. Moreover, MPF-dependent phosphorylation of Gwl promotes its further autophosphorylation required for its full activation (Yu et al., 2006). Mass spectrometry experiments identified several key residues from which some of them are required for $\mathrm{Gwl}$ activity. Results from mutagenesis analysis performed in Xenopus egg extracts identified 3 key residues critical for Gwl activity: Thr193/194, Thr206/207 and Ser883/875 (in Xenopus and human respectively) (Vigneron et al., 2011; Blake-Hodek et al., 2011). Because Thr193/194 and Thr206/207 lie within the minimal consensus site for Cdk kinases and these two residues are phosphorylated in vitro by Cdk1-cyclin B kinase, it has been proposed that Cdk1-Cyclin B may be the primary kinase responsible for the phosphorylation of these two sites. In this line, detailed experiments showed that Thr193/194 is a better substrate for MPF than Thr206/207. The Thr207 is a TPG motif which is highly unstable, and hence rapidly dephosphorylated (McCloy et al., 2015). Unlike these two residues, phosphorylation at Ser883/875 is essential for Gwl activity but does not fit with any kinase consensus site (Yu et al., 2006; Vigneron et al., 2011; Blake-Hodek et al., 2012). The hypothesis proposed by these studies is that first, MPF phosphorylates Thr193/194 and/or Thr206/207 and that subsequently either an intramolecular Gwl autophosphorylation or a Plk-1 dependent phosphorylation targets the phosphorylation at Ser883/875 to allow its full activity (Vigneron et al., 2011; Blake-Hodek et al., 2011).

Gwl is an atypical AGC kinase containing a long T-loop of about 500 amino acids that separates its $\mathrm{N}$-and $\mathrm{C}$-terminal catalytic domains. Experiments have been done to establish whether this central insert was critical for Gwl activity. From the two first studies developed in Xenopus egg extracts, none of the residue located inside this long insert appears to be essential for Gwl activity (Vigneron et al., 2011; Blake-Hodek et al., 2012). Nonetheless, very recent studies performed in HeLa cells provide evidence that both Ser90 and Ser453 in humans, Ser89 and Ser465 in Xenopus are required to maintain full Gwl activity (Della monica et al., 2015). Unfortunately, the identity of the kinases that phosphorylate these sites are still unknown.

Because phosphorylation of Gwl in these key residues is necessary for its activation at the G2-M transition, dephosphorylation of these same sites should be required for its inactivation. Clarity into the mechanisms responsible for dephosphorylation and inactivation of Gwl came from the identification of protein phosphatases involved in this pathway. Three different phosphatases PP1, PP2A-B55 and Fcp1 have been reported to play important roles in mitotic progression. During mitosis, PP1 is maintained inhibited by a direct phosphorylation on Thr320 by Cdk1-cyclin B. At the metaphase/anaphase transition, degradation of cyclin B results in a decrease of Cdk1-cyclin B activity thereby resulting in the autodephosphorylation at this residue and full activation of PP1 
(Wu et al., 2009). Additionally, PP2A-B55 is also inhibited at mitotic entry to ensure that Cdk1 substrates remain phosphorylated and it is subsequently reactivated at mitotic exit to induce the dephosphorylation of these substrates (Mochida et al., 2009; Schmitz et al., 2010). Finally, Fcp1 has been recently identified as another key mitotic regulator. Fcp1, also known as CTDP1: Carboxy-Terminal Domain, RNA polymerase II Subunit 1 was initially known as the phosphatase in charge of the dephosphorylation of the RNA polymerase II large subunit carboxyl terminal domain during initiation of transcription in eukaryotic cells and acts as crucial transcription regulator (Suh et al., 2005). Fcp1-depleted cell extracts maintained cyclin B stability and MPM2 signals as well as Wee1, Cdc20 and USP44 phosphorylations suggesting that Fcp1 plays a crucial role in mitotic exit (Visconti et al., 2012).

Interestingly, new data support that some of them would, at least in part, mediate mitotic exit through the dephosphorylation of Gwl. In this line, OA (Okadaic acid)-mediated PP2A inhibition or siRNA co-depletion of B55 $\alpha$ and $\delta$ from HeLa cells significantly blocked dephosphorylation of Gwl on its critical site Thr194 (Hegarat et al., 2014). In parallel of this study, three independent studies have involved PP1 as the major phosphatase required for Gwl inactivation. One of the three works has been developed either in human cell extracts using co-immunoprecipitation or in human cells using PLA (Proximity Ligation Assay) approaches. This study showed that human Gwl strongly interact with the catalytic subunit PP1 $\beta$. In the same line, authors showed that purified PP1 $\alpha$ promotes dephosphorylation of Gwl suggesting that there is some redundancy between Alpha and Beta isoforms of PP1. Additionally, using a PP1-disrupting peptide (PDP3) that enhance PP1 phosphatase activity, the authors showed that this peptide induces partial dephosphorylation of $\mathrm{Gwl}$ in prometaphase cell extracts (Rogers et al., 2016).

The two others groups performed their studies in Xenopus egg extracts. Both found that inhibition or depletion of PP1 strongly maintains full phosphorylation of Gwl upon Cdk1 kinase inhibition (Roscovitin) or inactivation (cyclin B degradation) (Heim et al., 2015; Ma et al., 2016). Because Ser875/883 is one of the critical residues, corresponding to the autophosphorylation site required for $\mathrm{Gwl}$ activation, these works have examined this key residue. Both groups showed that removal of PP1 strongly affect the dephosphorylation of $\mathrm{Gwl}$ on the autophosphorylation site Ser875 thereby maintaining full activity upon Cdk1 inactivation. These observations are consistent with the data published by Kornbluth's and Peng's labs showing that depletion of PP1 prevents the dephosphorylation of mitotic substrates and that removal of endogenous Pnuts (Phosphatase 1 nuclear targeting subunit 1), a potent inhibitor of PP1, disrupts metaphase II arrest (Wu et al., 2009; Fischer et al., 2014).

Depletion of PP1 stabilizes the Ser875 autophosphorylating site of Gwl but only partially affects the dephosphorylation at Thr194, the T-loop motif of this kinase targeted by PP2A-B55 (Hegarat et al., 2014; Ma et al., 2016).

To determine whether PP2A-B55 controls the dephosphorylation of $\mathrm{Gwl}$, either an hyperactive form of $\mathrm{Gwl}$ or a thiophosphorylated Arpp19 protein were added in Xenopus egg extracts, in order to inhibitPP2A-B55. Under these conditions, dephosphorylation of Gwl on Thr194 and Ser875 was assessed by either the use of specific antibodies (Ma et al., 2016) or by analysis of the Gwl gel migration shift (Heim et al., 2015). Despite the fact that MPF was inactivated by degradation of cyclin B, the inhibition of PP2A-B55 resulted in the delay of the dephosphorylation of Gwl in both activatory sites and the maintenance of a partial mitotic state. Interestingly, reactivation of PP2A-B55 by depletion of Arpp19 in these extracts promoted a rapid dephosphorylation of both sites and complete mitotic exit indicating that both residues are dephosphorylated by PP2A-B55 when it is fully activated (Ma et al., 2016) (Fig. 4).
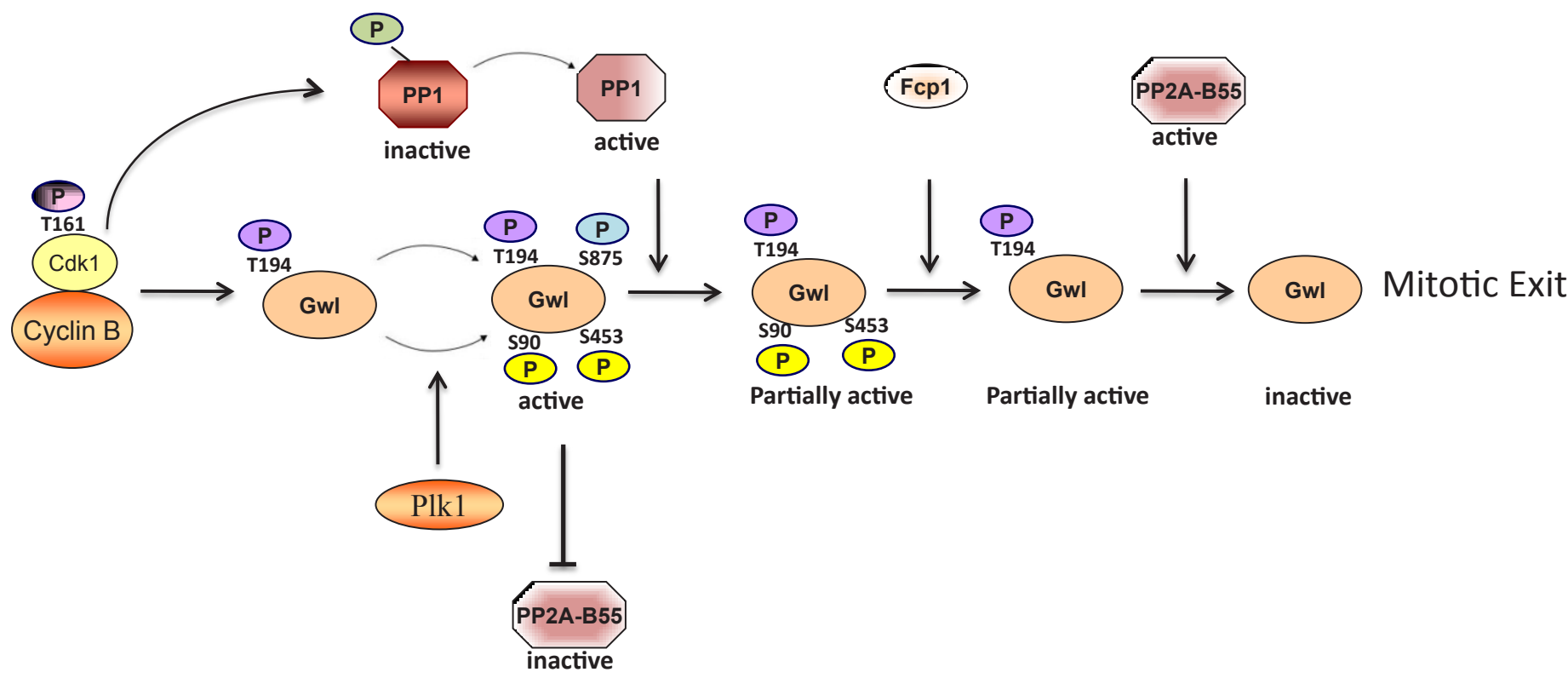

Fig. 4. Phosphatases promoting inactivation of Greatwall activity. At metaphase/anaphase transition, degradation of cyclin B allows autodephosphorylation of PP1 on Thr320. Then, PP1 is activated thereby promoting dephosphorylation of Gwl on its Ser875 activating site. At the same time Fcp1 dephosphorylates Ser90 and Ser453 to decrease Gwl activity. This induces a partial reactivation of PP2A-B55 which dephosphorylates Gwl on its Thr194 site allowing full Gwl inactivation and mitotic exit. 
Fcp1 has been described to reverse some Cdk-dependent phosphorylations (Visconti et al., 2012). To test whether Fcp1 is required to control the dephosphorylation of Gwl and thus promoting mitotic exit, experiments using co-immunoprecipitations showed that endogenous Fcp1 and Gwl are transiently associated during mitotic exit. Moreover, Fcp-1 depleted cells treated with RO-3306 (a potent inhibitor of Cdk1-cyclin B activity) to promote mitotic exit, delayed the dephosphorylation of Gwl at both Ser90 and Ser453. In addition, Ser90A mutation negatively affects Gwl kinase activity of $\mathrm{Gwl}$ and in vitro experiments showed that active Fcp1 purified protein induces dephosphorylation of $\mathrm{Gwl}$ at both Ser90 and Ser453. Altogether, these data indicate that in HeLa cells, Fcp1 is required for dephosphorylation of Gwl at Ser90 and Ser453 and its inactivation at mitotic exit (Della Monica et al., 2015).

The latest works published led us to believe that in mammalian cells and in Xenopusoocytes the ballet of the phosphatases involves different signalling pathways. In a first step, degradation of mitotic cyclinB allows the initiation of the inactivation of Cdk1 kinase. This promotes the reactivation of PP1 by autodephosphorylation at Thr320 inhibitory site. Consequently, the dephosphorylation of PP1 initiates dephosphorylation (at Ser875) and partial inactivation of Gwl thereby promoting the reactivation of PP2A-B55. Actually, both PP2A-B55 and PP1 and, may be, other phosphatases like PP2AB56 or Fcp1 are necessary for the complete dephosphorylation of mitotic substrates including $\mathrm{Gwl}$ and to mitotic exit.

\section{The dephosphorylation and the turning off Arpp19/ENSA}

Beside Gwl dephosphorylation, Fcp1 could also participate in Arpp19/ENSA dephosphorylation. In this line, removal of Fcp1 from HeLa cells had maintained Gwl-dependent phosphorylation of Arpp19/ENSA on Ser62/67 suggesting that Fcp1 might participated in mitotic exit (Hegarat et al., 2014). Meanwhile, the dephosphorylation of Endos (the Drosophila homologue of Arpp19/ ENSA) was also analysed independently by another group. This biochemical study found that the phosphorylated form of Endos (pEndos) strongly binds active PP2A-B55 and acts as a unfair competitive substrate of PP2A-B55, since its dephosphorylation is very slow it prevents dephosphorylation of others Cdk1-cyclin B substrates and thereby controls mitotic exit. (Williams et al., 2014; Gharbi-Ayachi etal., 2010; Mochida et al., 2010). In this context, Gwl phosphorylates Endos, which in turn associates with and inhibits PP2A-B55. When the degradation of cyclin B begins, Cdk1 and Gwl activities decrease, resulting in a drop of phosphorylation of Endos. PP2A-B55 becomes less and less inhibited and increasingly active thereby allowing dephosphorylation of both Arpp19/ ENSA inhibitor and mitotic substrates and promoted mitotic exit (Williams et al., 2014).

\section{DNA damage and cancer}

PP2A is known to be a major tumour suppressor in multiple malignancies including breast cancer (Eichhorn et al., 2009). Importantly, a massive study of genomic alterations in breast cancer identified deletions on PPP2R2A (encoding B55 $\alpha$ ) as one of the most frequent chromosomal alterations in luminal breast tumours (Curtis et al., 2012). Since Gwl is essential for promoting mitotic entry by indirectly inhibiting PP2A-B55 and because evidences are available supporting the idea that PP2A-B55 is a tumour sup- pressor, it was important to determine whether together $\mathrm{Gwl}$ and PP2A-B55 are involved in the cancerous process. With this aim, studies have been developed in order to understand whether Gwl participate in a significant extent to tumorogenesis pathway.

Importantly, our recent results show that Gwl overexpression promotes cell transformation in different immortalised cell lines (NIH3T3 and MCF10A) and in primary human fibroblasts. Besides its transforming capacities, Gwl overexpression increases "in cellulo" invasive properties and promotes an increase of tumour growth "in vivo". Interestingly, the effect of Gwl overexpression in cell proliferation, migration and invasion is mostly induced by the hyperactivation of Akt through the hyperphosphorylation of the activating residue of this kinase, Ser473. Despite the fact that the sole reported role of Gwl is the inhibition of PP2A-B55 through Arpp19/ ENSA phosphorylation, we clearly demonstrated that phosphorylation of Akt at Ser473 residue is not mediated by this pathway (Fig. 5). In contrast, Gwl overexpression stimulates the degradation of PHLPP, the phosphatase responsible for AKT dephosphorylation on Ser473 (Vera et al., 2015). Finally, we showed that Gwl is commonly overexpressed in colorectal cancer patients. Other results of a role of Gwl in head and neck squamous cell carcinoma (HNSCC) have also been reported. In this line, it has been shown that Gwl overexpression correlates with poor patient survival and tumour recurrence after cisplatin treatment suggesting a putative role of Gwl on chemotherapy response in patients with HNSCC (Wang et al., 2014). In addition, Arpp19 expression is increased in human hepatocellular carcinoma (HCC) and its levels correlate with tumour size. To reveal the role of Arpp19 in HCC, silencing Arpp19 by RNAi in human hepatocarcinoma cells was used. Results showed a lowered cell growth rate and retarded colony formation in these cells after Arpp19 knockdown (Song et al., 2015).

Besides its role in cancerogenesis, Gwl appears to be also involved in the DNA damage checkpoint. The G2 DNA damage checkpoint controls the G2-M transition to maintain genome integrity. Evidence of a direct role of Gwl activity in DNA damage recovery has been reported in Xenopus egg extract model (Peng et al., 2010). Indeed, studies in Xenopus egg extracts showed that $\mathrm{Gwl}$ is inhibited in response to DNA damage and that it is required to DNA damage recovery. Reactivation of Gwl during DNA damage recovery is dependent on its direct association to and its phosphorylation by Plx1 (Peng et al., 2011). In order to understand if this role is conserved in higher eukaryotes, experiments have been performed in cells by either overexpressing a hyperactive form of Gwl (K72M) or by knocking down Gwl during DNA damage recovery. Overexpression of Gwl-K72M accelerates checkpoint recovery and mitotic entry, conversely Gwl depleted cells delayed entry into mitosis after DNA damage.

Finally, either Arpp19-depleted or ENSA-depleted cells delayed checkpoint recovery indicating that this recovery may depend on the activating phosphorylation of Gwl substrates (Wong et al., 2016). Collectively, these results indicate that Gwl-Arpp19/ENSAPP2A-B55 pathway is required for DNA-damaged cells to maintain G2-arrest and genome integrity.

In summary, both Cdk1-cyclin B and Greatwall kinases control the entry into mitosis by phosphorylating several mitotic regulators and inhibiting the phosphatase that promote these dephosphorylations. However, the question is still open as to what the essential role of $\mathrm{Gwl}$ is in and outside of mitosis? Indeed, in embryonic cells the main role Gwl starts after NEBD (Nuclear Envelop BreakDown). 


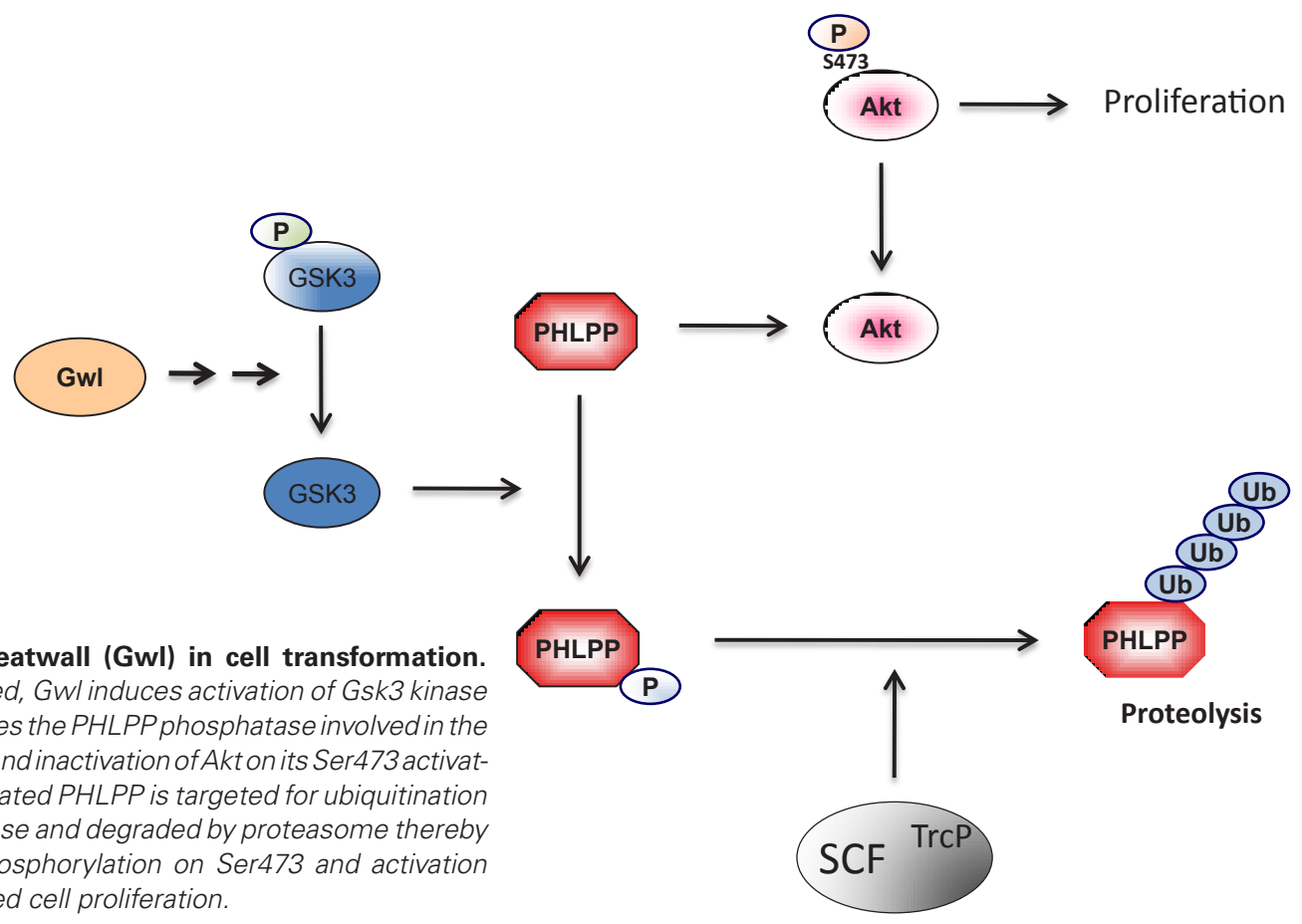

By contrast, in other systems such as mouse oocytes or Xenopus oocytes extracts, Gwl is essential for entry into metaphase II or for its maintenance. To explain this discrepancy, an intriguing possibility could be that threshold activities of phosphatase (PP2A-B55) and kinase (Cdk1-cyclin B) are not equal in both models used. An alternative possibility is that Arpp 19 and Ensa can be activated by both Gwl and Cdk1-cyclin B, the latter occurring when the former is absent or expressed less. Finally, in a context outside of mitosis, Gwl controls Akt phosphorylation in a Arpp19/ENSA independent pathway. In this line, it becomes critical to identify other substrates which could explain the different roles of Gwl.

\section{Acknowledgements}

Research in our group is supported by Ligue Nationale Contre le Cancer (LNCC) and ANR: Grant numbers ANR-10-BLAN-12-07. PR was supported by LNCC (Ph.D fellowship); KH was supported by a postdoctoral fellowship from Labex Epigenmed and LS was supported by a doctoral fellowship from Labex Epigenmed.

\section{References}

ABRIEU A, BRASSAC T, GALAS S, FISHER D, LABBÉ JC, DORÉE M (1998). The Polo-like kinase PIx1 is a component of the MPF amplification loop at the G2/Mphase transition of the cell cycle in Xenopus eggs. J Cell Sci. 111: 1751-1757.

ADHIKARI D, DIRIL MK, BUSAYAVALASA K, RISAL S, NAKAGAWA S, LINDKVIST R, SHEN Y, COPPOLA V, TESSAROLLO L, KUDO NR, KALDIS P, LIU K (2014). Mastl is required for timely activation of $\mathrm{APC} / \mathrm{C}$ in meiosis I and Cdk1 reactivation in meiosis II. J Cell Biol. 206: 843-853.

BETTENCOURT-DIAS, M., GIET, R., SINKA, R., MAZUMDAR, A., LOCK, W.G., BALLOUX, F., ZAFIROPOULOS, P.J., YAMAGUCHI, S., WINTER, S., CARTHEW, R.W., COOPER M, JONES D, FRENZ L, GLOVER DM (2004). Genome-wide survey of protein kinases required for cell cycle progression. Nature 432: 980-987.

BONTRON S, JAQUENOUD M, VAGA S, TALAREK N, BODENMILLER B, AEBERSOLD R, DE VIRGILIO C (2013). Yeast endosulfines control entry into quiescence and chronological life span by inhibiting protein phosphatase 2A. Cell Rep. 3:16-22.

BLAKE-HODEK KA, WILLIAMS BC, ZHAO Y, CASTILHO PV, CHEN W, MAO Y,

YAMAMOTO TM, GOLDBERG ML (2012). Determinants for activation of the atypical AGC kinase Greatwall during M phase entry. Mol Cell Biol. 32: 1337-1353.

BURGESS, A., VIGNERON, S., BRIOUDES, E., LABBÉ, J.-C., LORCA, T., CASTRO, $A(2010)$ Loss of human Greatwall results in G2 arrest and multiple mitotic defects due to deregulation of the cyclin B-Cdc2/PP2A balance Proc. Natl. Acad. Sci. USA 107, 12564-12569.

BRUINSMA W, MACUREK L, FREIRE R, LINDQVIST A, MEDEMA RH (2014) Bora and Aurora-A continue to activate Plk1 in mitosis. J Cell Sci. 127801-127811

CASTILHO PV, WILLIAMS BC, MOCHIDA S, ZHAO Y, GOLDBERG ML (2009) The M phase kinase Greatwall (Gwl) promotes inactivation of PP2A/B55delta, a phosphatase directed against CDK phosphosites. Mol Biol Cell. 20: 4777-4789.

CASTRO A, BERNIS C, VIGNERON S, LABBÉ JC, LORCA T (2005) The anaphasepromoting complex: a key factor in the regulation of cell cycle. Oncogene 24: 314-325.

CHAN EH, SANTAMARIA A, SILLJÉ HH, NIGG EA (2008) PIk1 regulates mitotic Aurora A function through betaTrCP-dependent degradation of hBora. Chromosoma. 117: 457-469.

CHICA N, ROZALÉN AE, PÉREZ-HIDALGO L, RUBIO A, NOVAK B, MORENO S (2016) Nutritional Control of Cell Size by the Greatwall-Endosulfine-PP2A·B55 Pathway. Curr Biol 26: 319-330.

CUNDELL MJ, BASTOS RN, ZHANG T, HOLDER J, GRUNEBERG U, NOVAK B, BARR FA (2013) The BEG (PP2A-B55/ENSA/Greatwall) pathway ensures cytokinesis follows chromosome separation. Mol Cell. 52: 393-405.

CURTIS C, SHAH SP, CHIN SF, TURASHVILI G, RUEDA OM, DUNNING MJ, SPEED D, LYNCH AG, SAMARAJIWA S, YUAN Y, GRÄF S, HA G, HAFFARI G, BASHASHATI A, RUSSELL R, MCKINNEY S; METABRIC GROUP, LANGERØD A, GREEN A, PROVENZANO E, WISHART G, PINDER S, WATSON P, MARKOWETZ F, MURPHY L, ELLIS I, PURUSHOTHAM A, BØRRESEN-DALE AL, BRENTON JD, TAVARÉ S, CALDAS C, APARICIO S (2012) The genomic and transcriptomic architecture of 2,000 breast tumours reveals novel subgroups. Nature. 483: 346-352.

DEIBLER RW, KIRSCHNER MW (2010) Quantitative reconstitution of mitotic CDK1 activation in somatic cell extracts. Mol Cell. 37: 753-767.

DELLA MONICA R, VISCONTI R, CERVONE N, SERPICO AF, GRIECO D (2015) Fcp1 phosphatase controls Greatwall kinase to promote PP2A-B55 activation and mitotic progression. Elife. 14: e10399

DUPRÉ A, BUFFIN E, ROUSTAN C, NAIRN AC, JESSUS C, HACCARD O (2013) The phosphorylation of ARPP19 by Greatwall renders the auto-amplification of 
MPF independently of PKA in Xenopus oocytes. J Cell Sci. 126: 3916-3926.

DUPRÉ A, DALDELLO EM, NAIRNAC, JESSUS C, HACCARD O (2014) Phosphorylation of ARPP19 by protein kinase A prevents meiosis resumption in Xenopus oocytes. Nat Commun. 5: 3318-3322.

EICHHORN PJ, CREYGHTON MP, BERNARDS R (2009) Protein phosphatase 2A regulatory subunits and cancer. Biochim Biophys Acta 1795: 1-15.

FISHER LA, WANG L, WU L, PENG A (2014) Phosphatase 1 nuclear targeting subunit is an essential regulator of M-phase entry, maintenance, and exit. $J$ Biol Chem. 289: 23745-23752.

FUNG TK, MA HT, POON RY (2007) Specialized roles of the two mitotic cyclins in somatic cells: cyclin A as an activator of $\mathrm{M}$ phase-promoting factor Mol Biol Cell. 18: 1861-1873.

FURUNO N, DEN ELZEN N, PINES J (1999) Human cyclin A is required for mitosis until mid prophase. J Cell Biol. 147: 295-306.

GHARBI-AYACHIA, LABBEJC, BURGESSA, VIGNERONS, STRUBJM, BRIOUDES E, VAN-DORSSELAER A, CASTRO A ANDLORCA T (2010) The substrate of Greatwall kinase, Arpp19, controls mitosis by inhibiting protein phosphatase 2A. Science 330: 1673-1677.

GOLDSTONE S, PAVEY S, FORREST A, SINNAMON J, GABRIELLI B (2001) Cdc25-dependent activation of cyclin A/cdk2 is blocked in G2 phase arrested cells independently of ATM/ATR. Oncogene 20: 921-932.

JUANES MA, KHOUEIRY R, KUPKA T, CASTRO A, MUDRAK I, OGRIS E, LORCA T and PIATTI S (2013) Budding yeast Greatwall and Endosulfines control activity and spatial regulation of PP2ACdc55 for timely mitotic progression. Plos Genetics 9, e1003575.

KALASZCZYNSKAI, GENG Y, IINOT, MIZUNO S, CHOIY, KONDRATIUK I, SILVER DP, WOLGEMUTH DJ, AKASHI K, SICINSKI P (2009) Cyclin A is redundant in fibroblasts but essential in hematopoietic and embryonic stem cells. Cell 138: 352-365.

KISHIMOTO T (2015) Entry into mitosis: a solution to the decades-long enigma of MPF. Chromosoma 124: 417-428.

KNOBLICH JA, LEHNER CF (1993) Synergistic action of Drosophila cyclins A and $B$ during the G2-M transition. EMBO J. 12: 65-74.

KUMAGAI A, DUNPHY WG (1996) Purification and molecular cloning of Plx1, a Cdc25-regulatory kinase from Xenopus egg extracts. Science. 273: 1377-1380.

HARA M, ABE Y, TANAKA T, YAMAMOTO T, OKUMURA E, KISHIMOTO T (2012) Greatwall kinase and cyclin B-Cdk1 are both critical constituents of M-phasepromoting factor. Nat Commun. 3: 1059-1064.

HÉGARAT N, VESELY C, VINOD PK, OCASIO C, PETER N, GANNON J, OLIVER AW, NOVÁK B, HOCHEGGER H (2014) PP2A/B55 and Fcp1 regulate Greatwall and Ensa dephosphorylation during mitotic exit. PLoS Genet. 10: e1004004.

HEIM A, KONIETZNY A, MAYER TU (2015) Protein phosphatase 1 is essential for Greatwall inactivation at mitotic exit. EMBO Rep. 16: 1501-1510.

LAOUKILI J, KOOISTRA MR, BRÁS A, KAUW J, KERKHOVEN RM, MORRISON A, CLEVERS H, MEDEMA RH (2005) FoxM1 is required for execution of the mitotic programme and chromosome stability. Nat Cell Biol. 7: 126-136.

LAOUKILI J, ALVAREZ M, MEIJER LA, STAHL M, MOHAMMED S, KLEIJ L, HECK AJ, MEDEMA RH (2008) Activation of FoxM1 during G2 requires cyclin A/Cdkdependent relief of autorepression by the FoxM1 N-terminal domain. Mol Cell Biol. 28: 3076-3087.

LI C, ANDRAKE M, DUNBRACK R, ENDERS GH (2010) A bifunctional regulatory element in human somatic Wee1 mediates cyclin A/Cdk2 binding and Crm1dependent nuclear export. Mol Cell Biol 30: 116-130.

LI YH, KANG H, XU YN, HEO YT, CUI XS, KIM NH, OH JS (2013) Greatwall kinase is required for meiotic maturation in porcine oocytes. Biol Reprod. 89: 53-59.

LINDQVIST A, RODRÍGUEZ-BRAVO V, MEDEMA RH (2009) The decision to enter mitosis: feedback and redundancy in the mitotic entry network. J Cell Biol. 185: 193-202.

LORCA T., BERNIS C., VIGNERON V., BURGESS A., BRIOUDES E., LABBÉ JC., CASTRO A (2010) Constant regulation of the MPF amplification loop and of the Greatwall/PP2A pathway is required for metaphasell-arrest and for a correct entry into first embryonic cell cycle. J Cell Sci 123: 2281-2291.

MAS, VIGNERONS, ROBERTP, STRUB JM, CIANFERANIS, CASTRO A, LORCAT (2016) Greatwall dephosphorylation and inactivation upon mitotic exit is triggered by PP1. J Cell Sci. 129: 1329-1339.
MACŮREK L, LINDQVIST A, LIM D, LAMPSON MA, KLOMPMAKER R, FREIRE R, CLOUIN C, TAYLOR SS, YAFFE MB, MEDEMA RH (2008) Polo-like kinase-1 is activated by aurora A to promote checkpoint recovery. Nature. 455:119-123.

MATTHEWS LM, EVANS JP (2014) $\alpha$-endosulfine (ENSA) regulates exit from prophase I arrest in mouse oocytes. Cell Cycle. 13:1639-1649.

MCCLOY RA, PARKER BL, ROGERS S, CHAUDHURI R, GAYEVSKIY V, HOFFMAN NJ, ALI N, WATKINS DN, DALY RJ, JAMES DE, LORCA T, CASTRO A, BURGESS A (2015) Global Phosphoproteomic Mapping of Early Mitotic Exit in Human Cells Identifies Novel Substrate Dephosphorylation Motifs. Mol Cell Proteomics. 14: 2194-2212.

MITRA J, ENDERS GH (2004) Cyclin A/Cdk2 complexes regulate activation of Cdk1 and Cdc25 phosphatases in human cells. Oncogene. 23: 3361-3367.

MOCHIDA S, IKEO S, GANNON J, HUNT T (2009) Regulated activity of PP2A-B55 delta is crucial for controlling entry into and exit from mitosis in Xenopus egg extracts. EMBO J 28: 2777-2785.

MOCHIDAS (2014) Regulation of $\alpha$-endosulfine, an inhibitor of protein phosphatase 2A, by multisite phosphorylation. FEBS J. 281: 1159-1169.

MOCHIDA S, MASLEN SL, SKEHEL M and HUNT T (2010) Greatwall phosphorylates an inhibitor of protein phosphatase $2 \mathrm{~A}$ that is essential for mitosis. Science 330: 1670-1673.

OH, J.S., SUSOR, A., SCHINDLER, K., SCHULTZ, R.M., CONTI, M (2013) Cdc25A activity is required for the metaphase II arrest in mouse oocytes. J Cell Sci. 126: 1081-1085.

OKUMURA E, MORITA A, WAKAI M, MOCHIDA S, HARA M, KISHIMOTO T (2014) Cyclin B-Cdk1 inhibits protein phosphatase PP2A-B55 via a Greatwall kinaseindependent mechanism. J Cell Biol. 204: 881-889.

PENG A, WANG L, FISHER LA (2011) Greatwall and Polo-like kinase 1 coordinate to promote checkpoint recovery. J Biol Chem. 286: 28996-29004.

PENG A, YAMAMOTO TM, GOLDBERG ML, MALLER JL (2010) A novel role for greatwall kinase in recovery from DNA damage. Cell Cycle. 9:4364-4369.

ROGERS S, FEY D, MCCLOY RA, PARKER BL, MITCHELL NJ, PAYNE RJ, DALY RJ, JAMES DE, CALDON CE, WATKINS DN, CROUCHER DR, BURGESS A (2016) PP1 initiates the dephosphorylation of MASTL, triggering mitotic exit and bistability in human cells. J Cell Sci. 129: 1340-54.

ROSHAKAK, CAPPER EA, IMBURGIAC, FORNWALD J, SCOTT G, MARSHALLLA (2000) The human polo-like kinase, PLK, regulates cdc2/cyclin B through phosphorylation and activation of the cdc25C phosphatase. Cell Signal. 12: 405-411.

ROSSIO V, KAZATSKAYA A, HIRABAYASHI M, YOSHIDA S (2014) Comparative genetic analysis of PP2A-Cdc55 regulators in budding yeast Cell Cycle. 13 : 2073-2083.

SCHMITZ MH, HELD M, JANSSENS V, HUTCHINS JR, HUDECZ O, IVANOVA E, GORIS J, TRINKLE-MULCAHYL, LAMONDAI, POSERI, HYMANAA, MECHTLER K, PETERS JM, GERLICH DW (2010) Live-cell imaging RNAi screen identifies PP2A-B55alpha and importin-beta1 as key mitotic exit regulators in human cells. Nat Cell Biol. 12: 886-893.

SEKI A, COPPINGER JA, JANG CY, YATES JR, FANG G (2008) Bora and the kinase Aurora a cooperatively activate the kinase Plk1 and control mitotic entry. Science. 320: 1655-1658.

SHI Y (2009) Serine/threonine phosphatases: mechanism through structure. Cell. 139: 468-484

SONG H, PAN J, LIU Y, WEN H, WANG L, CUI J, LIU Y, HU B, YAO Z, JI G (2014) Increased ARPP-19 expression is associated with hepatocellular carcinoma. Int $J$ Mol Sci. 16: 178-192.

SUH MH, YEP, ZHANG M, HAUSMANN S, SHUMAN S, GNATTAL, FU J (2005) Fcp1 directly recognizes the $C$-terminal domain (CTD) and interacts with a site on RNA polymerase II distinct from the CTD. Proc Natl Acad Sci USA. 102: 17314-17319.

VERAJ, LARTIGUE L, VIGNERON S, GADEA G, GIRE V, DEL RIO M, SOUBEYRAN I, CHIBON F, LORCA T, CASTRO A (2015) Greatwall promotes cell transformation by hyperactivating AKT in human malignancies. Elife. 27: e10115.

VIGNERON S, BRIOUDES E, BURGESS A, LABBE JC, LORCAT, CASTRO A (2009) Greatwall maintains mitosis through regulation of PP2A. EMBO J 28: 2786-2793.

VIGNERON S, GHARBI-AYACHI A, RAYMOND AA, BURGESS A, LABBÉ JC, LABESSE G, MONSARRAT B, LORCA T, CASTRO A (2011) Characterization of the mechanisms controlling Greatwall activity. Mol Cell Biol. 31: 2262-2275.

VISCONTI R, PALAZZO L, DELLA MONICA R, GRIECO D (2012) Fcp1-dependent 


\section{S. Vigneron et al.}

dephosphorylation is required for $\mathrm{M}$-phase-promoting factor inactivation at mitosis exit. Nat Commun. 3: 894.

VOETSE, WOLTHUIS RM (2010) MASTL is the human orthologue of Greatwall kinase that facilitates mitotic entry, anaphase and cytokinesis. Cell Cycle 9: 3591-3601

VOETS E, WOLTHUIS R (2015) MASTL promotes cyclin B1 destruction by enforcing Cdc20-independent binding of cyclin B1 to the APC/C. Biol Open. 4: 484-495.

VON STETINAJR, TRANGUCHS, DEYSK, LEE LA, CHAB, DRUMMOND-BARBOSA D (2008) alpha-Endosulfine is a conserved protein required for oocyte meiotic maturation in Drosophila. Development 135: 3697-3706.

WANG L, LUONG VQ, GIANNINI PJ, PENG A (2014) Mastl kinase, a promising therapeutic target, promotes cancer recurrence Oncotarget. 5: 11479-11489.

WANG P, PINSON X, ARCHAMBAULT, V (2011) PP2A-twins is antagonized by greatwall and collaborates with polo for cell cycle progression and centrosome attachment to nuclei in Drosophila embryos. PLoS Genet. 7: e1002227.

WILLIAMS BC, FILTER JJ, BLAKE-HODEK KA, WADZINSKI BE, FUDA NJ, SHALLOWAY D, GOLDBERG ML (2014) Greatwall-phosphorylated Endosulfine is both an inhibitor and a substrate of PP2A-B55 heterotrimers. Elife. 11: e01695.

WONG PY, MA HT, LEE HJ, POON RY (2016) MASTL(Greatwall) regulates DNA damage responses by coordinating mitotic entry after checkpoint recovery and APC/C activation. Sci Rep. 6: 22230.

WU JQ, GUO JY, TANG W, YANG CS, FREELCD, CHENC, NAIRNAC, KORNBLUTH $S$ (2009) PP1-mediated dephosphorylation of phosphoproteins at mitotic exit is controlled by inhibitor-1 and PP1 phosphorylation. Nat Cell Biol. 11: 644-651.

YU J, FLEMING S.L, WILLIAMS B, WILLIAMS E.V, LI Z, SOMMA P, RIEDER C.L, GOLDBERG M.L (2004) Greatwall kinase: a nuclear protein required for proper chromosome condensation and mitotic progression in Drosophila. J. Cell Biol. 164: 487-492.

YU J, ZHAO Y, LI Z, GALAS S, GOLDBERG ML (2006) Greatwall kinase participates in the Cdc2 autoregulatory loop in Xenopus egg extracts. Mol Cell. 22: 83-91.

ZHAO Y, HACCARD O, WANG R, YU J, KUANG J, JESSUS C, GOLDBERG M.L (2008) Roles of Greatwall kinase in the regulation of cdc25 phosphatase. Mol. Biol. Cell 19: 1317-1327. 


\section{Further Related Reading, published previously in the Int. J. Dev. Biol.}

Control of timing of embryonic M-phase entry and exit is differentially sensitive to CDK1 and PP2A balance Mohammed El Dika, Damian Dudka, Claude Prigent, Jean-Pierre Tassan, Malgorzata Kloc and Jacek Z. Kubiak Int. J. Dev. Biol. (2014) 58: 767-774

Identification of the sperm motility-initiating substance in the newt, Cynops pyrrhogaster, and its possible relationship with the acrosome reaction during internal fertilization

Toshihiko Watanabe, Hideo Kubo, Shinya Takeshima, Mami Nakagawa, Manami Ohta, Saori Kamimura, Eriko Takayama-Watanabe, Akihiko Watanabe, and Kazuo Onitake

Int. J. Dev. Biol. (2010) 54: 591-597

Signalling molecules involved in mouse bladder smooth muscle cellular differentiation

Benchun Liu, Dongxiao Feng, Guiting Lin, Mei Cao, Yuet Wai Kan, Gerald R. Cunha and Laurence S. Baskin

Int. J. Dev. Biol. (2010) 54: 175-180

Nuclear reprogramming in zygotes

Chanchao Lorthongpanich, Davor Solter and Chin Yan Lim

Int. J. Dev. Biol. (2010) 54: 1631-1640

Faithful reprogramming to pluripotency in mammals - what does nuclear transfer teach us?

Julien Maruotti, Alice Jouneau and Jean-Paul Renard

Int. J. Dev. Biol. (2010) 54: 1609-1621

Gonad-stimulating substance-like molecule from the radial nerve of the sea cucumber Hideki Katow, Tomoko Katow and Akihiko Moriyama

Int. J. Dev. Biol. (2009) 53: 483-491

Cyclin B2/cyclin-dependent kinase1 dissociation precedes CDK1 Thr-161 dephosphorylation upon M-phase promoting factor inactivation in Xenopus laevis cell-free extract

Franck Chesnel, Franck Bazile, Aude Pascal and Jacek Z. Kubiak

Int. J. Dev. Biol. (2007) 51: 297-305

Remodeling of sperm chromatin induced in egg extracts of amphibians.

C Katagiri and K Ohsumi

Int. J. Dev. Biol. (1994) 38: 209-216

The influence of mouse sera, regenerating liver extracts and bacterial products on the abilities of different cells in vitro.

N Zarkovic, M Osmak, D Novak, N Lers and M Jurin

Int. J. Dev. Biol. (1991) 35: 239-249

Hormonal factors from the mammalian pineal gland interfere with cell development in Hydra.

W A Müller, C Bartsch, H Bartsch, I Maidonis and E Bayer

Int. J. Dev. Biol. (1998) 42: 821-824
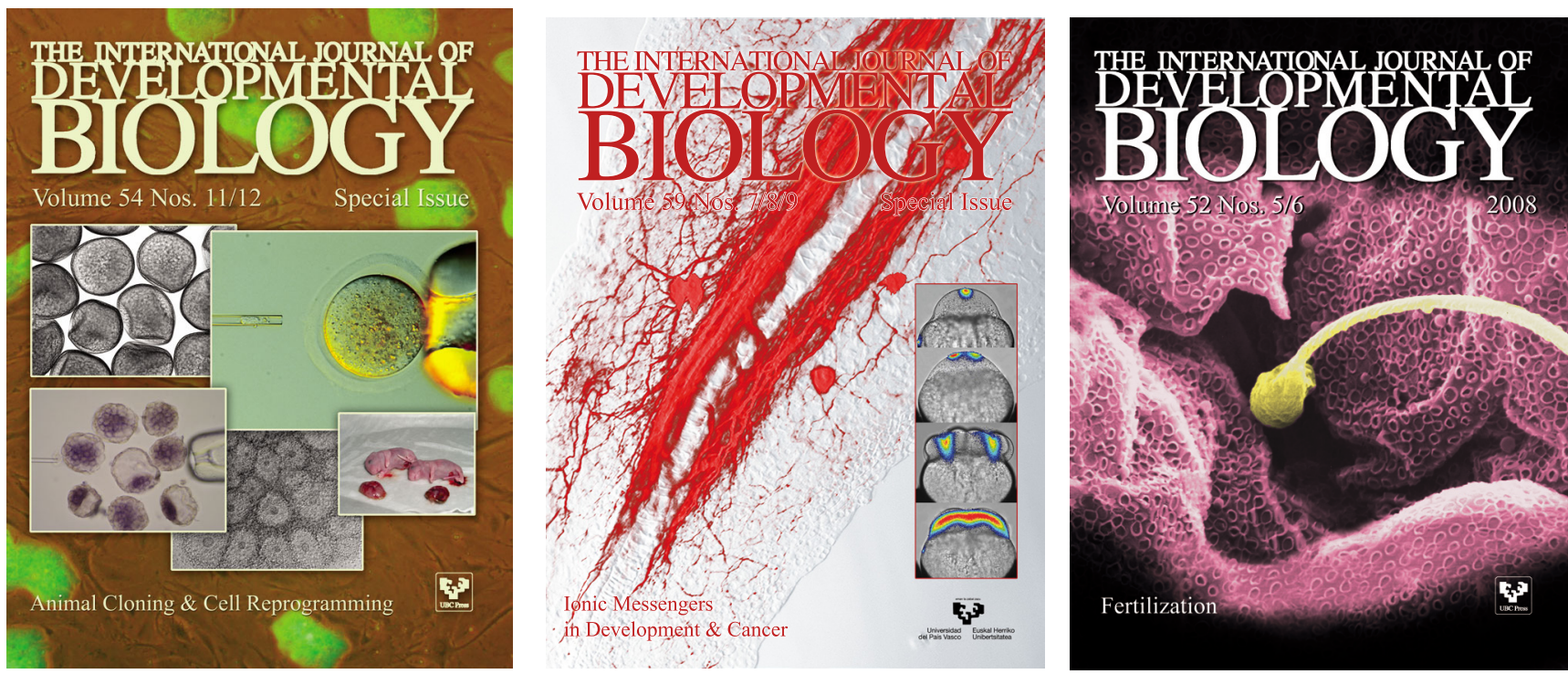